\title{
An All-Lag Rotating-Reference Correlator and its Efficient Implementation
}

\author{
Tung-Sang Ng, Kun-Wah Yip and Chin-Long Cheng \\ Department of Electrical and Electronic Engineering, The University of Hong Kong \\ Pokfulam Road, Hong Kong \\ Fax. $++852+25598738$ Tel.: $++852+28592710$ Email: $\{$ tsng, kwyip, clcheng $\} @$ eee.hku.hk
}

\begin{abstract}
This paper proposes an all-lag correlator that employs a rotating reference code sequence. The proposed correlator has an advantage over the previously proposed all-lag correlator, which employs a fixed reference code, in that correlation peaks remain at the same output positions regardless of update from newly arrived data samples. Therefore, the correlation information can be more conveniently manipulated in certain applications, and the posterior signal processing requirement can be reduced. Low-complexity implementation of the correlator using a recursive form is also presented. The degree of implementation complexity is of the order $N$, the reference-code length.
\end{abstract}

\section{INTRODUCTION}

One of the essential elements in the implementation of direct-sequence spread-spectrum (DSSS) systems is a correlator. Commonly used forms of correlators include serial correlators, parallel correlators and banks of serial correlators [1]-[9]. Recently, the authors [10] have introduced all-lag correlators, which correlate a stream of incoming data samples with $0,1, \cdots, N-1$ lags of a length$N$ reference code sequence and produces a stream of all-lag correlations at a rate equal to the rate of incoming data samples. Since more correlation information is provided by the all-lag correlator than other commonly used forms of correlators, some applications can take advantage of this additional correlation information. In addition, lowcomplexity implementation is made possible by using the recursive relationship derived in [10]. A review of the all-lag correlator is given in Section II.

Despite various advantages, the correlation peak of the all-lag correlator of [10] shifts from output to output as the correlation vector is updated by newly received data samples. Shifting of the peak position increases the subsequent signal processing requirement for extracting useful information provided by the all-lag correlation, and is inconvenient in certain applications. For example, in the estimation of the channel profile of a multipath channel in wireless communications, one is first required to locate the peak position among all correlator outputs. This peak position corresponds to the main path of the channel profile, and carries most of the signal energy. Shifting of the peak position requires one to continuously track the peak position using additional circuitry. On the other hand, if the peak position can be made to be fixed, one can read out the channel profile directly from the correlator outputs. Additional signal processing to continuously track the peak position is not required.

In order to overcome the peak-shifting problem, we propose in Section III a new all-lag correlator that employs rotating a reference code sequence. This correlator is referred to as the all-lag rotating-reference correlator, and is different from the one proposed in [10], in which a fixed reference code is used. The proposed correlator has correlation peaks remained at the same output positions regardless of update due to newly arrived data samples. Therefore, the correlation information can be more conveniently handled in certain applications, and the posterior signal processing requirement can be reduced. In Section IV, we derive a recursive form for the proposed correlator. Based on this recursive form, it is shown that an all-lag rotating-reference correlator can be implemented with low complexity. Finally, conclusion are presented in Section V.

\section{REVIEW OF THE ALL-LAG CORRELATOR}

Let $\left\{d_{n}\right\}$ denote the stream of incoming data samples and $\left\{c_{0}, c_{1}, \cdots, c_{N-1}\right\}$ be the reference code sequence. The alllag correlator proposed in [10] generates a stream of all-lag correlation vectors, $\left\{\mathbf{r}_{n}^{\prime}\right\}$, every sampling instant, where $\mathbf{r}_{n}^{\prime}=$ $\left[r_{0, n}^{\prime}, r_{1, n}^{\prime}, \cdots, r_{N-1, n}^{\prime}\right]^{T}$ is given by

$$
\mathbf{r}_{n}^{\prime}=\mathbf{C} \mathbf{d}_{n} \text {. }
$$

In the expression, $r_{m, n}^{\prime}$ denotes the $m$ th even-correlation lag

This work was supported by the Hong Kong Research Grants Council and by the University Research Committee of The University of Hong Kong, Hong Kong. The authors are with The University of Hong Kong. Fax: ++ $852+25598738$. Tel.: ++ $852+28592710$. (Email: \{tsng, kwyip, clcheng\}@eee.hku.hk) 
with reference to $\left\{c_{0}, c_{1}, \cdots, c_{N-1}\right\}$ obtained at time $n$,

$$
\mathbf{C}=\left[\begin{array}{cccccc}
c_{0} & c_{1} & c_{2} & \cdots & c_{N-2} & c_{N-1} \\
c_{N-1} & c_{0} & c_{1} & \cdots & c_{N-3} & c_{N-2} \\
c_{N-2} & c_{N-1} & c_{0} & \cdots & c_{N-4} & c_{N-3} \\
\vdots & \vdots & \vdots & & \vdots & \vdots \\
c_{2} & c_{3} & c_{4} & \cdots & c_{0} & c_{1} \\
c_{1} & c_{2} & c_{3} & \cdots & c_{N-1} & c_{0}
\end{array}\right]
$$

is an $N \times N$ matrix, and

$$
\mathbf{d}_{n}=\left[d_{n-(N-1)}, d_{n-(N-2)}, \cdots, d_{n-1}, d_{n}\right]^{T}
$$

is a data vector containing $N$ most recent data samples. Correlation information in $\mathbf{r}_{l}^{\prime}$, when processed, can be extracted for various purposes. The information provided by $\mathbf{r}_{n}^{\prime}$ is useful when the DSSS signal is composed of periodic repetition of the reference code sequence without data modulation.

To illustrate the peak-shifting property of the all-lag correlation, we consider a specific application in which the correlation information is used for the acquisition of a DSSS signal. Assume the DSSS signal is a periodic replica of the reference code without data modulation. Based on the information contained in $\mathbf{r}_{n}^{\prime}$ at time $n$, acquisition can be declared at the lag position where the largest magnitude among $r_{m, n}^{\prime}, m=0,1, \cdots, N-1$, occurs, say, the $\tilde{m}$ th lag position. When a new data sample is obtained at time $n+1$, the correlation vector is updated and $\mathbf{r}_{n+1}^{\prime}$ is computed. At this time instant, the lag position where the peak occurs is $\tilde{m}$ -1 when $\tilde{m}>0$, or $N-1$ if $\tilde{m}=0$. When a further data sample is obtained, the corresponding peak position shifts up for one additional step. Consequently, the peak position rotates as the all-lag correlation vector is updated, and has a period of $N$ sampling intervals.

\section{THE ALL-LAG ROTATING-REFERENCE CORRELATOR}

Define an $N \times N$ shift matrix

$$
\mathbf{S}=\left[\begin{array}{cccccc}
0 & 0 & 0 & \cdots & 0 & 1 \\
1 & 0 & 0 & \cdots & 0 & 0 \\
0 & 1 & 0 & \cdots & 0 & 0 \\
0 & 0 & 1 & \cdots & 0 & 0 \\
\vdots & \vdots & \vdots & & \vdots & \vdots \\
0 & 0 & 0 & \cdots & 0 & 0 \\
0 & 0 & 0 & \cdots & 1 & 0
\end{array}\right] .
$$

This matrix performs an end-around rotation of a length- $N$ column vector. It is easy to show that

$$
\mathbf{S}^{N}=\mathbf{I}
$$

where $\mathbf{I}$ is the $N \times N$ identity matrix. Let $\mathbf{c}_{m}$ be the $m$ th column $^{1}$ of $\mathbf{C}, m \in\{0,1, \cdots, N-1\}$. That is,

$$
\mathbf{c}_{m}=\left[c_{m}, c_{m-1}, \cdots, c_{0}, c_{N-1}, c_{N-2}, \cdots, c_{m+1}\right]^{T} \text {. }
$$

It follows that

$$
\begin{aligned}
\mathbf{c}_{0} & =\mathbf{S} \mathbf{c}_{N-1} \\
\mathbf{c}_{m+1} & =\mathbf{S} \mathbf{c}_{m}, \quad m=0,1, \cdots, N-2 .
\end{aligned}
$$

The desired correlator is devised as follows. Refer to (1), and suppose that the square matrix used to generate the all-lag correlation result at time $n$ for data samples $d_{n-(N-1)}$, $d_{n-(N-2)}, \cdots, d_{n}$ is $\mathbf{C}=\left[\mathbf{c}_{0} \mathbf{c}_{1} \cdots \mathbf{c}_{N-1}\right]$, i.e., $\mathbf{r}_{n}^{\prime}=d_{n-(N-1)} \mathbf{c}_{0}+d_{n-(N-2)} \mathbf{c}_{1}+d_{n-(N-3)} \mathbf{c}_{2}+\cdots+d_{n-1} \mathbf{c}_{N-2}+d_{n} \mathbf{c}_{N-1}$. It is observed that $d_{n-(N-1)}, d_{n-(N-2)}, \cdots, d_{n-1}, d_{n}$ are associated with $\mathbf{c}_{0}, \mathbf{c}_{1}, \cdots, \mathbf{c}_{N-2}, \mathbf{c}_{N-1}$, respectively. At time $n+1$, a new data sample $d_{n+1}$ is obtained. The corresponding square matrix that is required to compute the updated all-lag correlation in order to maintain the same peak position at the output with data samples $d_{n-(N-2)}, d_{n-(N-3)}$, $\cdots, d_{n}, d_{n+1}$ is $\left[\mathbf{c}_{1} \mathbf{c}_{2} \cdots \mathbf{c}_{N-1} \mathbf{c}_{0}\right]$. That is, the correlation vector at time $n+1$ is given by

$$
\left(d_{n-(N-2)} \mathbf{c}_{1}+d_{n-(N-3)} \mathbf{c}_{2}+\cdots+d_{n-1} \mathbf{c}_{N-2}+d_{n} \mathbf{c}_{N-1}\right)+d_{n+1} \mathbf{c}_{0} .
$$

It is easy to show that

$$
\begin{aligned}
{\left[\mathbf{c}_{1} \mathbf{c}_{2} \cdots \mathbf{c}_{N-1} \mathbf{c}_{0}\right] } & =\left[\mathbf{S c}_{0} \mathbf{S c}_{1} \cdots \mathbf{S c}_{N-2} \mathbf{S c}_{N-1}\right] \\
& =\mathbf{S}\left[\mathbf{c}_{0} \mathbf{c}_{1} \cdots \mathbf{c}_{N-1}\right]
\end{aligned}
$$

Generalizing, we propose an all-lag rotating-reference correlator that generates at time $n$ an all-lag even-correlation vector $\mathbf{r}_{n}=\left[r_{0, n}, r_{1, n}, \cdots, r_{N-1 . n}\right]^{T}$, where, with appropriate alignment, $r_{m, n}$ is the $m$ th even-correlation lag. In addition, $\mathbf{r}_{n}$ is computed by

$$
\mathbf{r}_{n}=\mathbf{A}_{n} \mathbf{d}_{n}
$$

where $\mathbf{d}_{n}$ is a data vector given by (3), and $\mathbf{C}_{n}$ is an $N \times N$ matrix constructed by

$$
\begin{aligned}
\mathbf{A}_{0} & =\mathbf{C} \\
\mathbf{A}_{n+1} & =\mathbf{S A}_{n}, \quad n \geq 0 .
\end{aligned}
$$

It follows that

$$
\mathbf{A}_{n}=\mathbf{S}^{n} \mathbf{C}, \quad n \geq 0 \text {. }
$$

Note that $\mathbf{A}_{n}$ 's are dependent on the time index $n$ and are therefore time-variant. The leading row of $\mathbf{A}_{n}$ is the

1 Throughout this paper, the left uppermost element of a matrix is given an index $(0,0)$ rather than the usually assigned one $(1,1)$. 
reference code at time $n$, and can be easily shown to be

$$
\left[c_{n \bmod N}, c_{(n+1) \bmod N}, \cdots, c_{(n+N-1) \bmod N}\right] \text {. }
$$

It is apparent that the reference code at time $n+1$ is an endaround rotation of the one at time $n$, and hence the name given to the all-lag rotating-reference correlator. Since $\mathbf{S}^{N}=$ $\mathbf{I}$, the correlation result $\mathbf{r}_{n}$ generated by an all-lag rotatingreference correlator is the same as $\mathbf{r}_{n}^{\prime}$ generated by an all-lag correlator of [10] when $n$ is a multiple of $N$. That is,

$$
\mathbf{r}_{n}=\mathbf{r}_{n}^{\prime}, \quad n=0, N, 2 N, \cdots
$$

\section{THE RECURSIVE FORM AND IMPLEMENTATION ASPECTS}

To implement $\mathbf{r}_{n}$ given by (9), one can use $N$ parallel correlators. Since each parallel correlator requires $N$ multiplications to generate each output, the implementation of an all-lag rotating correlator needs $N^{2}$ multiplications to generate an $\mathbf{r}_{n}$. It involves significant complexity. Therefore, we derive as follows a recursive relationship that enables reduced-complexity implementation of the correlator.

The recursive form for $\mathbf{r}_{n}$ is derived by expressing $\mathbf{r}_{n}$ in terms of $\mathbf{r}_{n-1}$, so that the updated $\mathbf{r}_{n}$ can be generated with a knowledge of the all-lag correlation vector $\mathbf{r}_{n-1}$ obtained at the previous sampling instant. Let $\mathbf{a}_{m . n}$ be the $m$ th column of $A_{n}$ at time $n$ where $m \in\{0,1, \cdots, N-1\}$. It follows that

$$
\mathbf{a}_{m, n}=\mathbf{S}^{n} \mathbf{c}_{m}=\mathbf{S}^{n+m} \mathbf{c}_{0} \text {. }
$$

From (13), it can be easily shown that

$$
\begin{aligned}
\mathbf{a}_{N-1, n} & =\mathbf{a}_{0 . n-1} \\
\mathbf{a}_{m-1 . n} & =\mathbf{a}_{n \cdot n-1}, \quad m=1,2, \cdots, N-1 .
\end{aligned}
$$

Since $\mathbf{A}_{n}=\left[\begin{array}{llll}\mathbf{a}_{0, n} & \mathbf{a}_{1, n} & \cdots & \mathbf{a}_{N-1, n}\end{array}\right]$, (9) can be expressed as

$$
\mathbf{r}_{n}=d_{n} \mathbf{a}_{N-1, n}+d_{n-1} \mathbf{a}_{N-2, n}+d_{n-2} \mathbf{a}_{N-3, n}+\cdots+d_{n-(N-1)} \mathbf{a}_{0, n} .
$$

Applying (14) to (15) gives

$$
\begin{aligned}
\mathbf{r}_{n}= & d_{n} \mathbf{a}_{N-1 . n}+\left(d_{n-1} \mathbf{a}_{N-1, n-1}+d_{n-2} \mathbf{a}_{N-2, n-1}+\cdots\right. \\
& \left.+d_{n-(N-1)} \mathbf{a}_{1, n-1}+d_{n-N} \mathbf{a}_{0, n-1}\right)-d_{n-N} \mathbf{a}_{0, n-1} .
\end{aligned}
$$

Since $\mathbf{a}_{0, n-1}=\mathbf{a}_{N-1 . n}$ as noted from (14), we arrive at the recursive relationship

$$
\mathbf{r}_{n}=\mathbf{r}_{n-1}+\left(d_{n}-d_{n-N}\right) \mathbf{a}_{N-1, n} .
$$

Also notice that $\mathbf{a}_{N-1 . n}$ can be generated by the recursive formula

$$
\mathbf{a}_{N-1 . n}=\mathbf{S a}_{N-1 . n-1}
$$

with $\mathbf{a}_{N-1.0}=\mathbf{c}_{N-1}$. Eqn. (17) indicates that $\mathbf{r}_{n}$ can be generated by using $N$ multiplications only. It is a substantial reduction of the complexity when compared to implementation using $N$ parallel correlators, which involves $N^{2}$ multiplications.

As (17) is a recursive relationship only, it is necessary to find the initial condition that makes (17) and (15) yield the same result. Repeated application of (17) for $N$ times followed by an application of (14) gives

$$
\begin{aligned}
\mathbf{r}_{n} & =\mathbf{r}_{n-N}+\left(d_{n}-d_{n-N}\right) \mathbf{a}_{N-1, n}+\left(d_{n-1}\right. \\
& \left.-d_{n-N-1}\right) \mathbf{a}_{N-2, n}+\cdots+\left(d_{n-(N-1)}-d_{n-(2 N-1)}\right) \mathbf{a}_{0, n} .
\end{aligned}
$$

Assume that signal samples, $d_{n}$ 's, are available only for $n=$ $1,2,3, \cdots$ and that we are only interested in generating $\mathbf{r}_{N}$, $\mathbf{r}_{N+1}, \mathbf{r}_{N+2}, \cdots$. Eqns. (19) and (15) become identical for $n=$ $N$ if $\mathbf{r}_{0}=\mathbf{0}$ and $d_{0}=d_{-1}=\cdots=d_{-(N-1)}=0$. Using a valid result of $\mathbf{r}_{N}$, one can compute $\mathbf{r}_{n}, n>N$, by (17). Note that intermediate results $\mathbf{r}_{1}, \mathbf{r}_{2}, \cdots, \mathbf{r}_{N-1}$ are not valid.

The number of arithmetic operations for each update can be easily observed from (17) to be 1 subtraction, $N$ additions, and $N$ multiplications. Fig. 1 depicts a recursive all-lag rotating-reference correlator that computes $\left\{\mathbf{r}_{n}\right\}$. It requires a length- $N$ shift register to store input data samples, an output buffer to store $N$ correlation results obtained for the previous sampling instant, and an end-around shift register to store the rotating reference code, a negator, $N$ multipliers and $N+1$ two-input adders. The implementation complexity is therefore of the order $N$. Prior to operation, the values stored in the input-data shift register and the output storage are initialized to zero, and the end-around shift register is loaded with the basic reference code $\mathbf{c}_{N-1}$.

In some applications, it may be sufficient to generate $M$ out of $N$ correlation results every sampling instant, where $M$ $<N$. The recursive relationship given by $(17)$ indicates that a reduced correlator can be obtained from a full implementation by directly deleting those unused branches. It follows that the reduced all-lag rotating-reference correlator comprises a length- $N$ shift register for storing input data, an $M$-element buffer for storing $M$ desired correlation results obtained at the previous sampling instant, a length- $N$ shift register for storing the rotating reference code, 1 negator, $M$ multipliers and $M+1$ two-input adders. Since the implementation complexity is usually determined by the number of multipliers and adders, it follows that the resultant implementation complexity of a reduced correlator is of the order $M$. Reduction in the implementation cost is obtained in comparison to a full implementation.

\section{CONCLUSIONS}

The all-lag rotating-reference correlator for generating $\left\{\mathbf{r}_{n}\right\}$ has been proposed and the corresponding recursive form 
has been derived. Based on the derived recursive form, it has been shown that the implementation complexity of an all-lag rotating-reference correlator is of the order $N$. It has also been found that the complexity can be reduced when only $M$ correlation lags $(M<N)$ are required, in which case a reduced correlator can be implemented with a complexity of the order $M$.

\section{REFERENCES}

[1] D. T. Magill and G. Edwards, "Digital matched filter ASIC," Proc. IEEE MILCOM'90, pp. 235-238, Sep. 30 - Oct. 3, 1990.

[2] R. S. Mowbray and P. M. Grant, "Simplified matched filter receiver designs for spread spectrum communications applications," IEE Electronics and Communication Engineering Journal, pp. 59-64, Apr. 1993.

[3] R. C. Dixon and J. S. Vanderpool, "Spread spectrum correlator," U. S. Pat. No. 5,022,047, Jun. 4, 1991.

[4] R. C. Dixon and J. S. Vanderpool, "Dual-threshold spread spectrum correlator," U. S. Pat. No. 5,719,900, Feb. 17, 1998.

[5] I. S. Carnes and D. L. Shollenberger, "CW Radar range measuring system," U. S. Pat. No. 5,793,327, Aug. 11, 1998.

[6] R. Price and P. E. Green, Jr., "A communication technique for multipath channels," Proc. IRE, vol. 46, pp. 555-570, Mar. 1958.

[7] M. Luise and R. Reggiannini, "Carrier recovery in all-digital modems for burst-mode transmission," IEEE Trans. Commun., vol. 43, pp. 1169-1178, Feb./Mar./Apr. 1995.

[8] U. Fawer, "A coherent spread-spectrum diversity receiver with AFC for multipath fading channels," IEEE Trans. Commun., vol. 42, pp. 1300-1311, Feb./Mar./Apr. 1994.

[9] A. Q. Hu, P. C. K. Kwok and T. S. Ng, "MPSK DS/CDMA carrier recovery and tracking based on correlation technique," IEE Electron. Lett., vol. 35, pp. 201-203, Feb. 1999.

[10] T. T. S. Ng. K.-W. Yip and C.-L. Cheng, "Recursive all-lag reference-code correlator and its efficient implementation," accepted for presentation at IEEE ISCAS 2000, May $28-31$, 2000, Geneva, Switzerland.

Fig. 1. The recursive all-lag rotating-reference correlator.

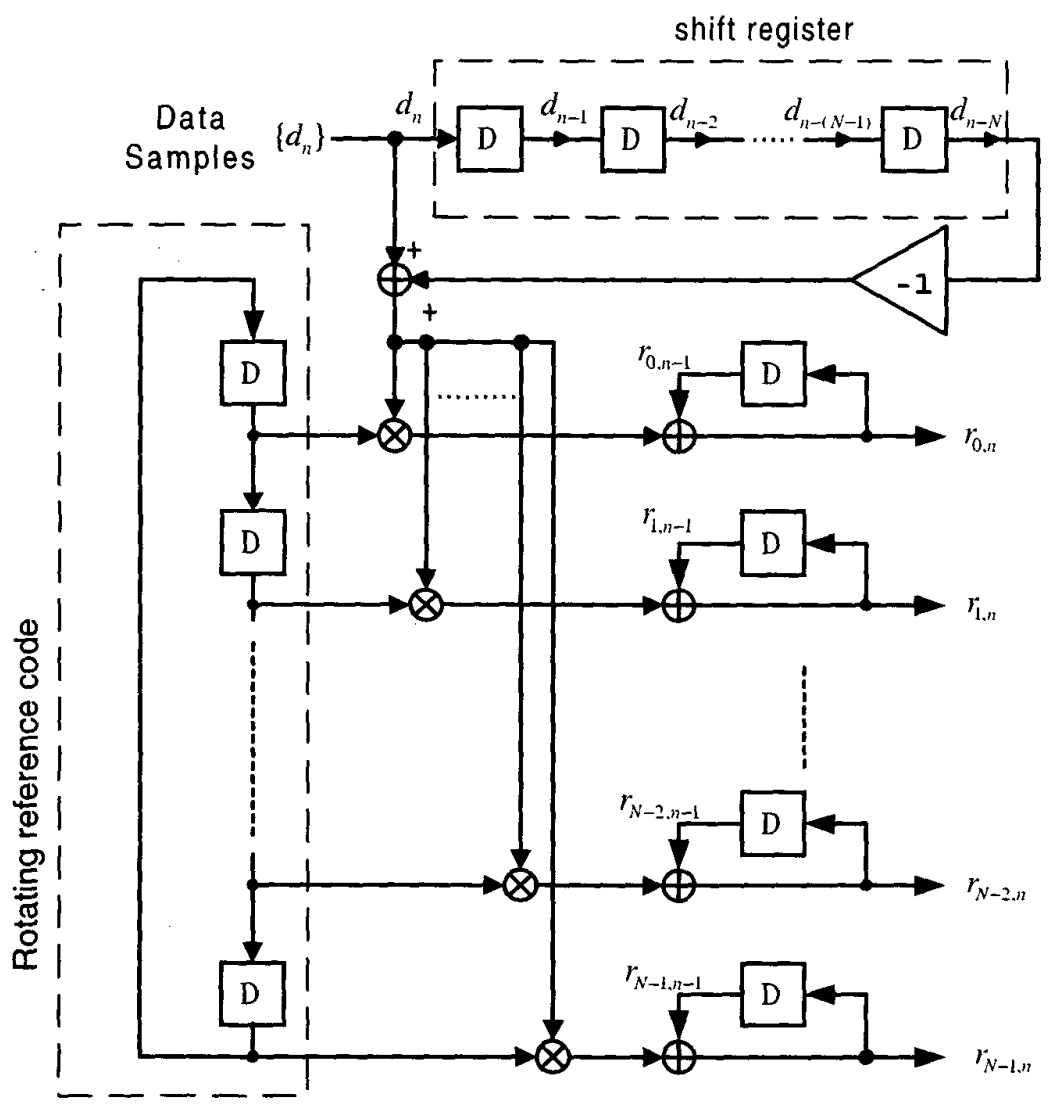

D storage unit 